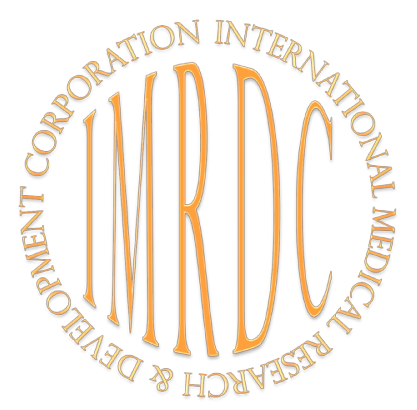

\title{
Application of Exogenous Luteinizing Hormone in IVF Patients of Late Reproductive Age
}

\author{
N. V. Protopopova ${ }^{1,2,3}$; E. B. Druzhinina ${ }^{1,2,3}$; N. A. Boldonova ${ }^{3}$; A. V. Labygina ${ }^{2}$; \\ Y. V. Mylnikova ${ }^{3}$; N. L. Sakhyanova ${ }^{3}$; L. I. Maschakevich ${ }^{3}$; K. V. Krylova ${ }^{3}$; N. A. Kurashova ${ }^{2 *}$ \\ ${ }^{1}$ Russian Medical Academy of Continuous Professional Education \\ ${ }^{2}$ Scientific Centre for Family Health and Human Reproduction Problems \\ ${ }^{3}$ Irkutsk Regional Clinical Hospital, Regional Perinatal Center \\ Irkutsk, the Russian Federation
}

\begin{abstract}
Background: Adecreased concentration of luteinizing hormone (LH) in women of late reproductive age contributes to disorder in adequate steroidogenesis and impairs oocyte/embryo quality, which results in fewer embryos suitable for cryopreservation, a lower implantation rate, and increased rate of early miscarriage. The purpose of our study was a retrospective analysis of the effectiveness of applying exogenous LH (urinary and recombinant) in ART programs in women of late reproductive age with previous IVF failures.

Methods and Results: We studied how adding exogenous LH (urinary and recombinant) from Days 2-3 and 6-7 of menstrual cycle in ovulatory stimulation affects the outcomes of in vitro fertilization cycles. The study included 324 women (mean age of $35.7 \pm 3.8$ years) receiving treatment for tubal-peritoneal infertility as part of IVF programs. All patients were divided into 4 groups depending on the stimulation day (Day 2-3 or Day 6-7) when exogenous LH-based inductor (human menopausal gonadotropin or recombinant LH) was added. In patients with a risk of "poor response," supplementing rLH at the initial stages of folliculogenesis (Day 2-3 of menstrual cycle) promotes optimization of oogenesis processes, obtaining a high percentage of good quality embryos $(78 \%)$ and increasing pregnancy rate (30\%). The good quality frozen embryos obtained in stimulation with recombinant LH and human menopausal gonadotropin added, had the same good quality and provided the PR comparable to transfer of fresh embryos.

Conclusion: It is possible that LH injection restores the microenvironment of developing follicles in women of late reproductive age, which is reasonable for the patients studied.(International Journal of Biomedicine. 2020;10(2):112-115.)
\end{abstract}

Key Words: in vitro fertilization • exogenous luteinizing hormone • pregnancy $\bullet$ embryo cryopreservation

\section{Abbreviations}

AMH, anti-Müllerian hormone; ART, assisted reproductive technology; CG, chorionic gonadotropin; FSN, follicle-stimulating hormone; GnRH, gonadotropin-releasing hormone; hMG, human menopausal gonadotropin; hCG, human CG; IVF, in vitro fertilization; LH, luteinizing hormone; MC, menstrual cycle; OHSS, ovarian hyperstimulation syndrome; PR, pregnancy rate; rFSH, recombinant FSH; rLH, recombinant LH.

\section{Introduction}

Preservation and restoration of the population's reproductive health are problems far from a permanent solution, yet are the most important medical challenge of national standing. The rate of infertile couples in Russia ranges from $8 \%$ to $20 \%$, which exceeds a $15 \%$ critical level as determined by WHO. ${ }^{(1-3)}$
The most important step in infertility treatment is to elaborate the ART methods and apply them to clinical practice. According to Russian Association of Human Reproduction, in 2017 the pregnancy rate in Russia was $33.9 \%$ per cycle, and $38.4 \%$ per embryo transfer in programs of in vitro fertilization (IVF). After age 35, the response of ovaries to ovulation stimulation is impaired, quality of the retrieved oocytes and their fertilization pattern is affected up to complete absence, 
and the nature and rate of embryo cleavage are transformedall of which finally leads to worse IVF results. ${ }^{(4,5)}$

Ovarian stimulation is the basis of assisted reproduction programs, which is thought to be optimal if one succeeds in retrieving a minimum of 7 preovulatory follicles. Maturation of 5-6 or less than 5 follicles is regarded as a suboptimal or "poor" response; it is found in nearly a third of the women of late reproductive age (over 35 years). ${ }^{(6)}$ Inadequate ovarian response leads to decreased IVF effectiveness.

In normal $\mathrm{MC}$, in follicular phase first half, the follicle development implies FSH-dependent growth; in the second half, LH-dependent growth begins when a dominant follicle is selected. ${ }^{(1)}$ Regimens with GnRH agonists and antagonists successfully solve the problem of LH early peak; use of rFSH devoid of LH activity leads to a significant decrease in the concentration of endogenous LH. On the other hand, in patients of late reproductive age we noted a decreased number of functional LH-receptors, which may lead to lower ovarian response. ${ }^{(4,7)}$

Depletion of the LH concentration contributes to disorder in adequate steroidogenesis and impairs the oocyte/ embryo quality; this results in a lower implantation rate, fewer embryos suitable for cryopreservation, and increased rate of early miscarriage. ${ }^{(8)}$ For the above reasons, the absolute or functional insufficiency of LH to improve the effectiveness of IVF calls for supplemental administration of exogenous LH preparations in stimulation cycles, such as highly purified HMG and rLH as well. ${ }^{(9-11)}$

The purpose of our study was a retrospective analysis of the effectiveness of applying exogenous LH in ART programs in women of late reproductive age with previous IVF failures.

\section{Materials and Methods}

We examined 324 women receiving treatment for tubal -peritoneal infertility as part of IVF programs in the ART Department of Irkutsk Regional Clinical Hospital. All women signed an informed consent for use of their personal data. All patients were comparable with respect to their medical history and clinical data, and their laboratory and ultrasound parameters. Their average age was $35.7 \pm 3.8$ years; they had reduced ovarian reserve (number of antral follicles $6.3 \pm 3.3$ ) and previous IVF failures, which was the predisposing cause of poor ovarian response during stimulation. The exclusion criteria were as follows: severe somatic pathology, concentration of functional sperm below $30 \%$.

We divided all patients into 4 groups depending on the stimulation day (Day 2-3 or Day 6-7) when exogenous LHbased inductor (hMG or rLH) was added. Gonadotropin doses and duration of stimulation were determined individually, taking into account the past medical history, age and dynamic ultrasound data. We stopped the rLH injections 2 days prior to the hCG trigger. ${ }^{(12)}$ We assessed the embryo stage parameters, pregnancy rate, "poor response," and OHSS.

For transvaginal ovarian puncture, we used a standard technique 36 hours after injection of a CG ovulatory dose with COOK double-lumen needles. Follicles were flushed with Flushing Medium buffer solution ("ORIGIO") in an amount not exceeding the amount of the retrieved follicular fluid. For cultivation of gametes and embryos, and for freezing and thawing of embryos, we used the ORIGIO medium line (MediCult Media), four-well trays "Nunc," and the $\mathrm{CO}^{2}-$ incubator ThermoForma, according to the manufacturer's recommendations. The applied freezing method was vitrification. We selected embryos for cryopreservation on Day 2-5 of the cultivation based on "good quality" standard criteria; the thawed embryos were transferred 2 hours after thawing. The embryos were transferred using a standard technique.

The studied patients were distributed as follows:

Group 1A included 123 patients: hMG was added on Day 2-3 of rFSH stimulation

Group 1B included 68 patients: hMG was added on Day 6-7 of rFSH stimulation

Group 2A included 70 patients: rLH was added on Day 2-3 of rFSH stimulation

Group 2B included 63 patients: rLH was added on Day 6-7 of rFSH stimulation

All the studied patients were comparable with respect to their medical history and clinical data, and their laboratory and ultrasound parameters (Table 1).

Table 1.

Description of initial parameters in patients with various stimulation protocols

\begin{tabular}{|l|c|c|c|c|c|}
\hline \multicolumn{1}{|c|}{ Variable } & $\begin{array}{c}\text { Group 1A } \\
(\mathrm{n}=123)\end{array}$ & $\begin{array}{c}\text { Group 1B } \\
(\mathrm{n}=68)\end{array}$ & $\begin{array}{c}\text { Group 2A } \\
(\mathrm{n}=70)\end{array}$ & $\begin{array}{c}\text { Group 2B } \\
(\mathrm{n}=63)\end{array}$ & $P$-value \\
\hline Age, years & $36.1 \pm 3.9$ & $35.5 \pm 3.6$ & $35.5 \pm 5.5$ & $35.6 \pm 3.7$ & $\geq 0.05$ \\
\hline $\begin{array}{l}\text { MC duration, } \\
\text { days }\end{array}$ & $28.9 \pm 6.1$ & $28.4 \pm 2.1$ & $28.6 \pm 4.3$ & $28.4 \pm 4.5$ & $\geq 0.05$ \\
\hline $\begin{array}{l}\text { Duration of } \\
\text { infertility, years }\end{array}$ & $7.6 \pm 4.2$ & $8.1 \pm 4.2$ & $9.3 \pm 5.3$ & $9.2 \pm 5.2$ & $\geq 0.05$ \\
\hline $\begin{array}{l}\text { Primary } \\
\text { infertility }\end{array}$ & $37 / 30.1 \%$ & $21 / 30.9 \%$ & $25 / 35.7 \%$ & $19 / 30.2 \%$ & $\geq 0.05$ \\
\hline $\begin{array}{l}\text { Secondary } \\
\text { infertility }\end{array}$ & $86 / 69.9 \%$ & $47 / 69.1 \%$ & $45 / 64.3 \%$ & $44 / 69.8 \%$ & $\geq 0.05$ \\
\hline $\begin{array}{l}\text { Basal LH. } \\
\text { mIU/ml }\end{array}$ & $5.0 \pm 3.3$ & $5.2 \pm 2.8$ & $5.1 \pm 3.0$ & $5.3 \pm 3.2$ & $\geq 0.05$ \\
\hline $\begin{array}{l}\text { Basal FSH, } \\
\text { mIU/ml }\end{array}$ & $8.2 \pm 3.8$ & $7.8 \pm 2.8$ & $8.4 \pm 4.1$ & $7.9 \pm 4.2$ & $\geq 0.05$ \\
\hline $\begin{array}{l}\text { Basal AMH, } \\
\text { ng/ml }\end{array}$ & $1.9 \pm 1.7$ & $2.0 \pm 1.9$ & $1.7 \pm 1.6$ & $1.9 \pm 1.6$ & $\geq 0.05$ \\
\hline $\begin{array}{l}\text { Number of antral } \\
\text { follicles in } \\
\text { both ovaries }\end{array}$ & $6.0 \pm 2.9$ & $6.0 \pm 3.2$ & $5.8 \pm 3.1$ & $6.1 \pm 3.1$ & $\geq 0.05$ \\
\hline
\end{tabular}

The statistical analysis was performed using the statistical software STATISTICA 6.1 (StatSoft Inc., USA). The mean (M) and standard deviation (SD) were calculated. For data with normal distribution, inter-group comparisons were performed using Student's t-test. Mann-Whitney U test was used to compare means of variables not normally distributed. A probability value of $P<0.05$ was considered statistically significant 


\section{Results and Discussion}

The day of LH injection was chosen based on recommendations from a great deal of published data. It is known that $\mathrm{LH}$ begins to impose the stimulating effect on the growing follicles once they reach a minimum of $10 \mathrm{~mm}$ in diameter, i.e. by the 6th day of stimulation.

The analysis of the embryo stage in 4 LH-stimulation protocols shows that supplementing with hMG or $\mathrm{rLH}$ during the ovarian stimulation process at different phases of follicular maturation has various effects on folliculogenesis and oogenesis (Table 2).

Table 2.

Description of embryo stage basic parameters

\begin{tabular}{|l|c|c|c|c|c|}
\hline \multicolumn{1}{|c|}{ Variable } & $\begin{array}{c}\text { Group } \\
1 \mathrm{~A} \\
(\mathrm{n}=123)\end{array}$ & $\begin{array}{c}\text { Group } \\
1 \mathrm{~B} \\
(\mathrm{n}=68)\end{array}$ & $\begin{array}{c}\text { Group } \\
2 \mathrm{~A} \\
(\mathrm{n}=70)\end{array}$ & $\begin{array}{c}\text { Group } \\
2 \mathrm{~B} \\
(\mathrm{n}=63)\end{array}$ & $P$-value \\
\hline $\begin{array}{l}\text { Number of punc- } \\
\text { tured follicles }\end{array}$ & $5.0 \pm 2.9$ & $4.8 \pm 2.9$ & $4.5 \pm 3.1$ & $5.0 \pm 3.4$ & $P \geq 0.05$ \\
\hline $\begin{array}{l}\text { Number of } \\
\text { oocytes retrieved }\end{array}$ & $3.6 \pm 2.9$ & $3.6 \pm 2.9$ & $2.9 \pm 2.7$ & $4.0 \pm 3.1$ & $P_{2 \mathrm{~A}-2 \mathrm{~B}}=0.009$ \\
\hline $\begin{array}{l}\text { Number of } \\
\text { mature oocytes }\end{array}$ & $3.0 \pm 2.6$ & $3.0 \pm 2.4$ & $2.3 \pm 2.1$ & $3.1 \pm 2.6$ & $P \geq 0.05$ \\
\hline $\begin{array}{l}\text { Number of } \\
\text { immature oocytes }\end{array}$ & $0.5 \pm 0.7$ & $0.6 \pm 0.8$ & $0.4 \pm 0.8$ & $0.8 \pm 1.1$ & $\begin{array}{l}P_{P_{2 \mathrm{~A}-2 \mathrm{~B}}=}=0.049 \\
P_{1 \mathrm{~B}-2 \mathrm{~A}}=0.011\end{array}$ \\
\hline $\begin{array}{l}\text { Number of } \\
\text { degenerative } \\
\text { oocytes }\end{array}$ & $0.2 \pm 0.4$ & $0.1 \pm 0.3$ & $0.1 \pm 0.3$ & $0.1 \pm 0.3$ & $P \geq 0.05$ \\
\hline $\begin{array}{l}\text { Number of } \\
\text { unfertilized } \\
\text { oocytes }\end{array}$ & $0.8 \pm 0.9$ & $0.8 \pm 1.0$ & $0.5 \pm 0.8$ & $1.0 \pm 1.4$ & $\begin{array}{l}P_{1 \mathrm{AA}-2 \mathrm{~A}}=0.048 \\
P_{1 \mathrm{~A}-2 \mathrm{~B}}=0.016\end{array}$ \\
\hline $\begin{array}{l}\text { Number of } \\
\text { embryos obtained }\end{array}$ & $2.7 \pm 2.4$ & $2.8 \pm 2.3$ & $2.3 \pm 2.1$ & $2.9 \pm 2.3$ & $P \geq 0.05$ \\
\hline $\begin{array}{l}\text { Number of } \\
\text { good quality } \\
\text { embryos }\end{array}$ & $1.8 \pm 1.3$ & $2.0 \pm 2.5$ & $1.8 \pm 1.1$ & $2.0 \pm 1.8$ & $P \geq 0.05$ \\
\hline $\begin{array}{l}\text { Proportion of } \\
\text { good } \\
\text { quality embryos }\end{array}$ & $65.9 \%$ & $70.5 \%$ & $78.0 \%$ & $67.1 \%$ & $\begin{array}{l}P \\
P_{2 \mathrm{~A}-2 \mathrm{~B}}=0.034\end{array}$ \\
\hline $\begin{array}{l}\text { Embryo } \\
\text { cryopreservation }\end{array}$ & $16.3 \%$ & $19.1 \% 009$ \\
\hline $\begin{array}{l}\text { Number of frozen } \\
\text { embryos }\end{array}$ & $0.4 \pm 1.1$ & $0.4 \pm 0.9$ & $0.3 \pm 0.9$ & $0.6 \pm 1.7$ & $P \geq 0.05$ \\
\hline
\end{tabular}

In all groups, there were no statistical differences in the average number of punctured follicles, retrieved mature and degenerative oocytes, and the number of embryos obtained, including those of good quality. However, the smallest number of oocytes $(2.9 \pm 2.7)$, including immature oocytes $(0.4 \pm 0.8)$, was retrieved in Group 2A. This fact evidences that in $\mathrm{rFSH}$ stimulation with rLH added from Day 2-3, the growth of preovulatory follicles was more uniform than in other groups, where with a greater number of the oocytes retrieved, most of them were immature. Group 2A gave the lowest number of unfertilized oocytes $(0.5 \pm 0.8)$, a fact that can be attributed to this group having the smallest number of immature oocytes, and to better quality of oocytes retrieved as well. The proportion of good quality embryos in Group $2 \mathrm{~A}$ was the largest among the 4 groups $(78 \%, 65.9 ; 70.5$ and $67.1 \%$, respectively). Good quality extra embryos were frozen in $16.3 \%$ of cases in Group 1A, 19.1\% - in Group 1B, 14.3\% in Group 2A, and $15.9 \%$ - in Group 2B ( $P>0.05)$. The average number of frozen embryos in all groups was not statistically different.

In recent years there has been a trend toward selective transfer into the uterine cavity of one good quality embryo that has reached the blastocyst stage, in order to reduce the risk of multiple pregnancy and to improve pregnancy rates. However, this can hardly be related to women with reduced parameters of ovarian reserve who have the risk of "poor" response, when it is safer to transfer 3-day embryos, as their further development is not guaranteed. In our study, the number of embryos that reached the blastocyst stage in patients of the 4 groups was $4 \%, 3.7 \%, 3.8 \%$ and $4.7 \%$, respectively $(P>0.05)$.

In Group 2A, there were the highest pregnancy rates per stimulated cycle and per embryo transfer, and the rate of term births $(30 \%, 36.8 \%$, and $25.7 \%$, respectively), which counts in favor of the best quality of oocytes and embryos obtained, and the endometrium readiness for implantation in the protocol with rLH added on Day 2-3 of MC (Figure 1).

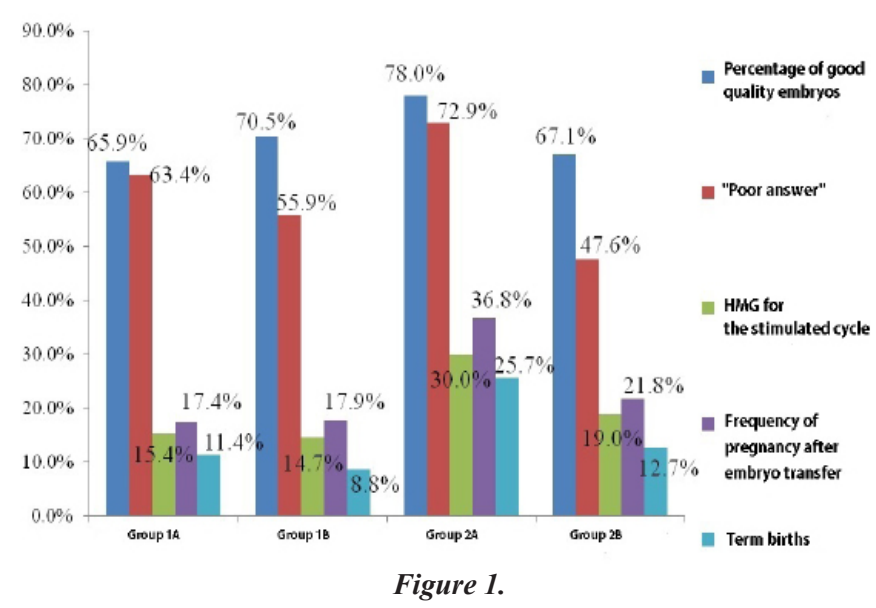

In Group 2A, such an IVF effectiveness parameter as "poor" response was noted at the highest level (72.9\%), which was further accompanied by retrieving the lowest number of oocytes in this group, but the greatest proportion of good quality embryos $(78 \%)$. In other words, the $2 \mathrm{~A}$ protocol did not increase the number of follicles punctured, but improved the quality of oocytes and embryos retrieved.

OHSS was registered at statistically the same low level in all groups $(3.3 \%, 5.9 \%, 5.7 \%$, and $3.2 \%)$. The OHSS low rate is conditioned by the initial low ovarian reserve in the patients studied.

Our study shows that supplementation of rLH at the initial stages of folliculogenesis is more physiological, since in the natural menstrual cycle, follicles grow in the presence of both endogenous FSH and LH from the early stages. On the other hand, the increased level with age of FSH, not LH, is noted in the early follicular phase. It is possible that $\mathrm{LH}$ injection restores the microenvironment of developing follicles 
in women of late reproductive age, which is reasonable for the patients studied.

At the next stage, we evaluated the effectiveness of transferring frozen embryos retrieved in 2 protocols (Table 3 ).

Table 3.

Effectiveness of transfer of frozen embryos retrieved in various stimulation protocols

\begin{tabular}{|l|c|c|c|}
\hline & $\begin{array}{c}\mathrm{rFSH}+\mathrm{HMG} \text { protocol } \\
(\mathrm{n}=98)\end{array}$ & $\begin{array}{c}\mathrm{rFSH}+\mathrm{rLH} \text { protocol } \\
(\mathrm{n}=46)\end{array}$ & $P$-value \\
\hline $\begin{array}{l}\text { Pregnancy rate, } \\
\text { case (\%) }\end{array}$ & $22(22.4 \%)$ & $13(28.3 \%)$ & $>0.05$ \\
\hline
\end{tabular}

The effectiveness of frozen embryo transfer does not depend on the stimulation protocol by which the frozen embryo was obtained, (i.e. whether the stimulation involved supplementation of recombinant or urine exogenous LH). In addition, PR was not statistically different in frozen and fresh embryo transfer (Figure 1).

As stated previously, in patients with the expected low ovarian response in a stimulated cycle it is safer to transfer 3-day embryos as their further development is not guaranteed. Therefore, for this cohort of patients in a stimulated cycle, we have to choose the most effective protocol that facilitates obtaining the best quality embryos (in our case, $\mathrm{rFSH}+\mathrm{rLH}$ from Day 2-3). Obtaining good quality extra embryos suitable for freezing is a good indicator of stimulation in these patients. In a non-stimulated cycle, only good quality frozen embryos are transferred, those that have passed another stage of selection-vitrification. Thus, the good quality frozen embryos obtained in stimulation with rLH and hMG added, have the same good quality and provide the PR comparable to transfer of fresh embryos.

In conclusion: In patients with a risk of "poor response," supplementing rLH at the initial stages of folliculogenesis (Day 2-3 of $\mathrm{MC}$ ) promotes optimization of oogenesis processes, obtaining a high percentage of good quality embryos $(78 \%)$ and increasing PR (30\%). Good frozen embryos obtained in stimulation with rLH and hMG added are of the same good quality, and pregnancy rates are not statistically different in transfer of frozen and fresh embryos.

\section{Competing Interests} interests

The authors declare that they have no competing

*Corresponding author: Nadezhda A. Kurashova, PhD, ScD. Scientific Centre for Family Health and Human Reproduction Problems, Irkutsk, the Russian Federation. E-mail: nakurashova@, yandex.ru

\section{Acknowledgements}

We thank the reproduction and embryology specialists of the Department of Assisted Reproductive Technologies for their service within the ART programs.

\section{References}

1. Boyarsky KIu, Gaidukov SN. [Folliculogenesis: from antral stage to ovulation (a review)]. Problemy reproduktsii [Russian Journal of Human Reproduction]. 2010;16(5):1323. [Article in Russian].

2. Ermolenko KS, Radzinsky VE, Rapoport SI. [State ofthe-art of realization of the fertility potential in the women of late reproductive age]. Klin Med (Mosk). 2016;94(1):10-16. [Article in Russian].

3. Labygina AV, Suturina LV, Kolesnikova LI, Darzhaev ZY, Dashiyev BG. [The reproductive health of native and outside the population of the eastern Siberia]. Zdravookhraneniye Rossiyskoy Federatsii. 2013;3:37-39. [Article in Russian].

4. Vuong TN, Phung HT, Ho MT. Recombinant folliclestimulating hormone and recombinant luteinizing hormone versus recombinant follicle-stimulating hormone alone during GnRH antagonist ovarian stimulation in patients aged $\geq 35$ years: a randomized controlled trial. Hum Reprod. 2015;30(5):1188-95. doi: 10.1093/humrep/dev038.

5. Protopopova NV, Dudakova VN, Druzhinina EB, Labygina AV, Boldonova NA, Krylova KV. The Effectiveness of In Vitro Fertilization Programs in Patients with Low AntiMüllerian Hormone Levels. Doctor.Ru. 2018;10(154):7-9. doi: 10.31550/1727-2378-2018-154-10-7-9

6. Younis JS, Ben-Ami M, Ben-Shlomo I. The Bologna criteria for poor ovarian response: a contemporary critical appraisal. J Ovarian Res. 2015;8:76. doi: 10.1186/s13048-015-0204-9.

7. Matorras R, Prieto B, Exposito A, Mendoza R, Crisol L, Herranz P, Burques S. Mid-follicular LH supplementation in women aged 35-39 years undergoing ICSI cycles: a randomized controlled study. Reprod Biomed Online. 2009;19(6):879-87.

8. Briggs R, Kovacs G, MacLachlan V, Motteram C, Baker HW. Can you ever collect too many oocytes? Hum Reprod. 2015;30(1):81-7. doi: 10.1093/humrep/deu272.

9. Pezzuto A, Ferrari B, Coppola F, Nardelli GB. LH supplementation in down-regulated women undergoing assisted reproduction with baseline low serum LH levels. Gynecol Endocrinol. 2010;26(2):118-24. doi: 10.3109/09513590903215516.

10. Mamedova NR, Nazarenko TA, Monakhova IV. Preparations containing the luteinizing hormone in assisted reproduction (a review). Problemy reproduktsii (Russian Journal of Human Reproduction). 2011;17(3):50-55.

11. Esteves SC, Alviggi C. The Role of LH in Controlled Ovarian Stimulation. Principles and Practice of Controlled Ovarian Stimulation in ART. 2015:171-196

12. Spencer JB, Browne AS, Copland SD, Session DR. Discontinuation of rLH two days before hCG may increase the number of oocytes retrieved in IVF. Reprod Biol Endocrinol. 2010;8:29. doi: 10.1186/1477-7827-8-29. 